\title{
Simulation of Gold Functionalization with Cysteine by Reactive Molecular Dynamics
}

\author{
Susanna Monti ${ }^{\dagger *}$, Vincenzo Carravetta ${ }^{\ddagger}$, Hans Ågren ${ }^{\dagger}$ \\ ${ }^{\dagger}$ Theoretical Chemistry and Biology, School of Biotechnology, KTH Royal \\ Institute of Technology, SE-10044 Stockholm, Sweden, ${ }^{\ddagger}$ CNR-IPCF, Institute \\ of Chemical and Physical Processes, via G. Moruzzi 1, I-56124 Pisa, Italy, and \\ ${ }^{\#}$ CNR-ICCOM , Institute of Chemistry of Organometallic Compounds, via G. \\ Moruzzi 1, I-56124 Pisa, Italy \\ E-mail: sapeptides@gmail.com
}

\section{Table of Contents}

Figure1S: Density Functional Theory Energy Optimizations Used to Create the Training Set for Parametrizing the Interaction of Cysteine with Gold

Figure2S: Energy differences of the configurations sampled during the ab initio MD simulation in relation to the starting structure. Comparison with the values obtained by means of single point calculations with the classical force field (ReaxFF - red dots).

Force Field Parameters: ffield file (format used in ReaxFF, serial version, LAMMPS and ADF)

Figure3S: Distance travelled by the Au add atom during the whole simulation time. The initial position has been placed in the origin of the reference system. Projections (light gold) on the different planes, namely $\mathrm{XY}, \mathrm{XZ}$ and $\mathrm{YZ}$, are displayed in order to give an idea of the relative positions of the atom. All the other gold atoms of the interface should be, in principle, in the XY plane (grey area).

Figure4S: Distributions of the minimum distances of cysteine and water atoms to the atoms of the support. The amino group remains far from the surface during the whole simulations time, whereas both the oxygens and sulfur atoms of the molecule are located at around $2.4 \mathrm{~A}$ from the slab. Water hydrogens are closer to gold than all the other atoms, whilst water oxygens are found at a longer separation in relation to the carboxyls' ones.

Figure 5S. Potential energy of the system as a function of the simulation time. 
Figure 1S. Density Functional Theory Energy Optimizations Used to Create the Training Set for Parametrizing the Interaction of Cysteine with Gold
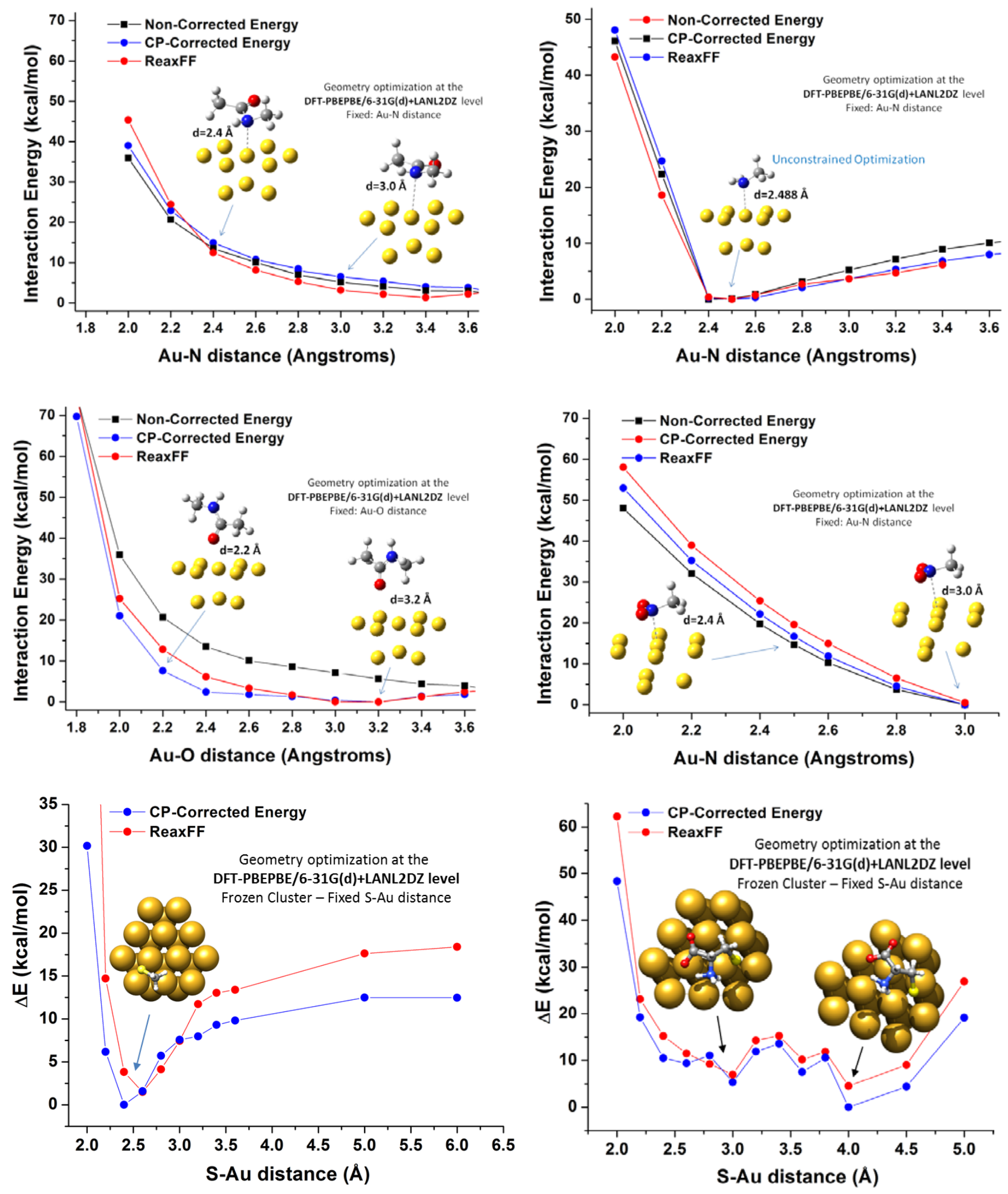


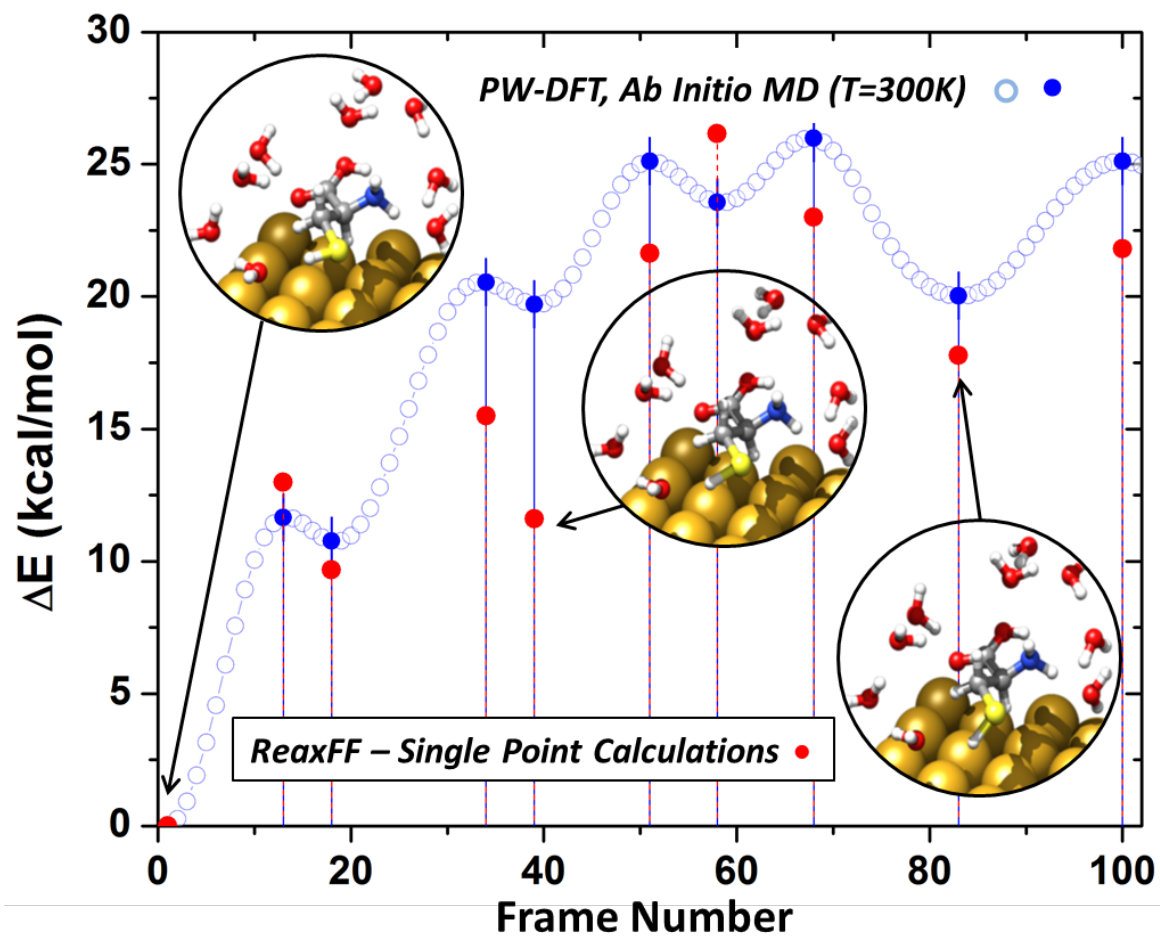

Figure 2S. Energy differences of the configurations sampled during the ab initio MD simulation in relation to the starting structure. Comparison with the values obtained by means of single point calculations with the classical force field (ReaxFF - red dots).

\section{Computational Details}

The gold slab (Au(111) surface) used for the ab initio molecular dynamics simulations was made of three layers of twenty gold atoms each and had dimensions of about $14.4 \times 10.0 \mathrm{~A}$ in the xy plane. The substrate was frozen, during all the calculations, at the crystal geometry (considering an Au lattice constant of $4.06 \AA ̊$ ). Periodic boundary conditions were applied in $x, y$ and $z$ directions (box in $z$ about $43 \AA$ ). The whole simulated system consisted of 101 atoms: $60 \mathrm{Au}, 1 \mathrm{CYS}$ (14 atoms), 9 waters (27 atoms).

Plane wave density functional theory calculations (PW-DFT) were performed by means of the Quantum Espresso package [1] with the PBE exchange-correlation functional [3] and ultra-soft pseudopotentials [2]. Kinetic energies and electron densities were cut at 50 and 400 Ry, respectively. The Brillouin zone integration was performed by means of Gaussian smearing with a width of 0.02 Ry. As a first step, the geometry of the molecule was energy minimized using the conjugate gradient (CG) method with a force convergence threshold of $0.05 \mathrm{eV} / \mathrm{A}$, then molecular dynamics simulations were carried out in the NVT ensemble at $\mathrm{T}=300 \mathrm{~K}$. Then, the systems was heated from 0 to $300 \mathrm{~K}$ in $0.3 \mathrm{ps}$, and subsequently simulated at that temperature for about $1.7 \mathrm{ps}$ with a time step of $0.24 \mathrm{fs}$.

Configurations were extracted during both the equilibration phase and production run by selecting relative minimum and maximum energy structures (Figure 2S). These were used to check the performance of the developed force field.

[1] P. Giannozzi et al. Quantum espresso: a modular and opensource software project for quantum simulations of materials, J. Phys. Condens. Matter 21, 395502 (2009)

[2] B. Hammer, L. B. Hansen, J. K. Norskov, Improved adsorption energetics within density-functional theory using revised perdew-burke-ernzerhof functionals Phys. Rev. B 59, 7413 (1999)

[3] D. Vanderbilt, Soft self-consistent pseudopotentials in a generalized eigenvalue formalism Phys. Rev. B, 41, 7892(1990) 


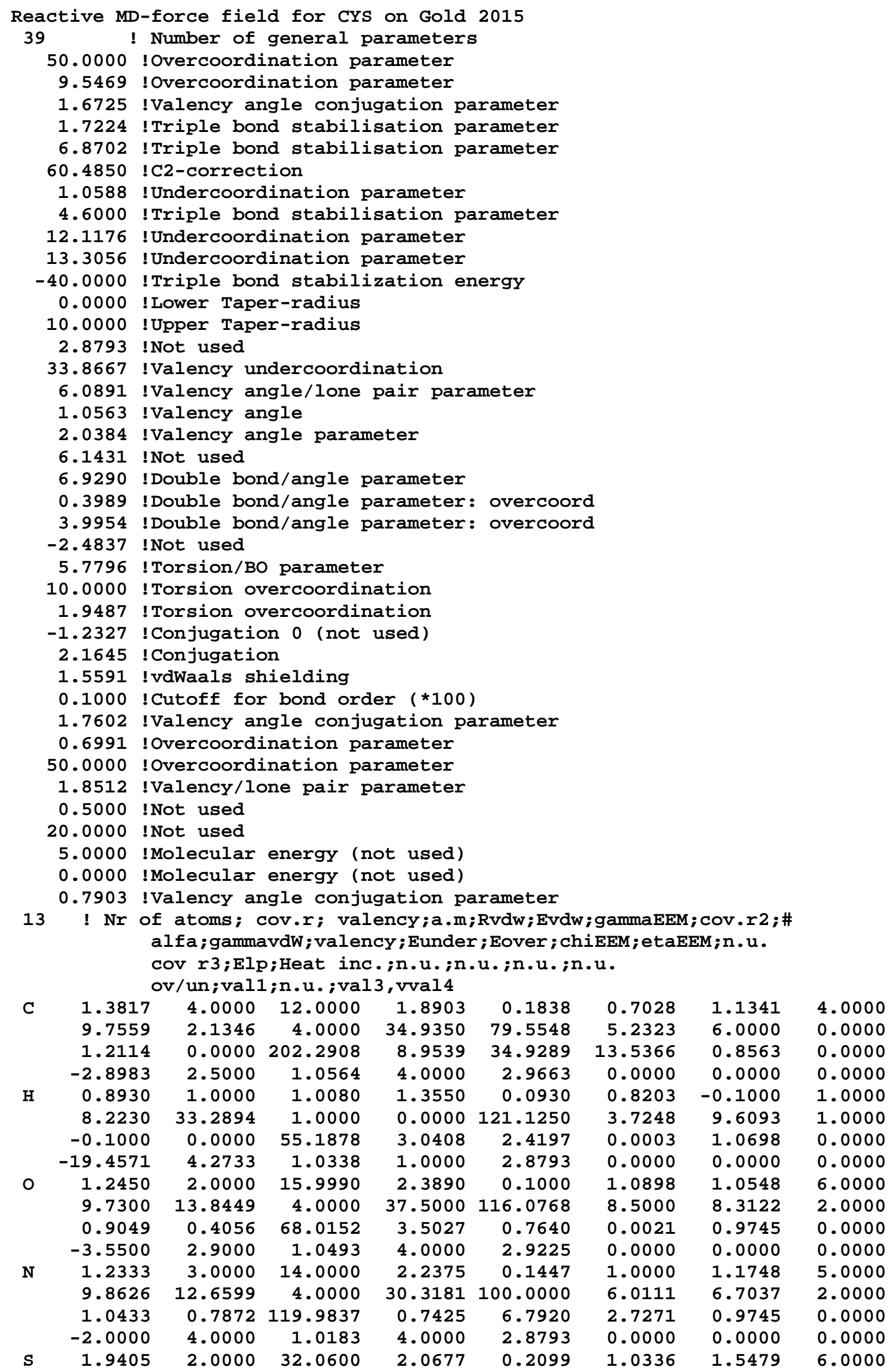




\begin{tabular}{|c|c|c|c|c|c|c|c|c|}
\hline & 9.9575 & 4.9055 & 4.0000 & 52.9998 & 112 . 1416 & 6.5000 & 8.2545 & 2000 \\
\hline & 1.4601 & 9.7177 & 71.1843 & 5.7487 & 23.2859 & 12.7147 & 0.9745 & 0.0000 \\
\hline & -11.0000 & 2.7466 & 1.0338 & 6.2998 & 2.8793 & 0.0000 & 0.0000 & 0.0000 \\
\hline & 1.8315 & 2.0000 & 24.3050 & 2.2464 & & 0.5020 & 1.0000 & \\
\hline & 10.9186 & 27.1205 & 3.0000 & 38.0000 & 000 & 499 & 5.6130 & 0.0000 \\
\hline & -1.3000 & & 127.9160 & & & & 0.0000 & \\
\hline & -1.0823 & 2.3663 & 1.0564 & & 2.9663 & 0.0000 & 0.0000 & 0000 \\
\hline & 1.5994 & & 30.9 & & & & & \\
\hline & 9.1909 & 14.9482 & 5.0000 & 0.0000 & 0.0000 & 1.6676 & 7.0946 & 1000 \\
\hline & -1.0000 & 25.0000 & 125.6 & & 21.4 & 15.1425 & 0.0000 & 0000 \\
\hline & -3.9294 & 3.4831 & 1.0 & & 793 & & 0.00 & \\
\hline $\mathrm{Na}$ & 1.8000 & 1.0000 & 22.9 & 2.8270 & 72 & 0.4000 & -1.0000 & 000 \\
\hline & 10.0 & & & & & -0.5 & 6.77 & \\
\hline & -1.0 & & 23.1 & 100. & & & 0.8 & 000 \\
\hline & -2.5 & 3.5 & & & & & 0.0 & \\
\hline Ti & 2.02 & & 47. & & & & 0.1 & 000 \\
\hline & 12.7 & 16.6 & & & & -1 . & 6.8 & \\
\hline & -1.0 & & 143. & & & & & \\
\hline & -15.0000 & 3.8 & 1.0 & 12. & & & 0.0 & 000 \\
\hline Cl & 1.7140 & 1.0 & 35. & & & & -1.0000 & \\
\hline & 11.5 & 10.1 & & & & & & \\
\hline & -1.0000 & 1.2 & 143. & & & & 0.8 & 000 \\
\hline & -10.20 & & & & & & & \\
\hline & 1.2100 & 1.0 & 18. & & & 000 & -0.1000 & 000 \\
\hline & & & & & & & 15. & \\
\hline & -1.0 & 35.0 & & & & & 0.00 & 000 \\
\hline & -7.3000 & 2.6 & & & & & 0.0000 & 000 \\
\hline $\mathrm{Au}$ & & & 196.9 & & & & & \\
\hline & 11.97 & 2.0 & & & & & 8.9 & 000 \\
\hline & -1.00 & & & & & & & \\
\hline & -24.7 & & & & & & 0.0000 & 000 \\
\hline & -0.1 & & & & & & -0.10 & \\
\hline & & & & & & & 1.5 & \\
\hline & & & -2.3700 & & & & 0.9745 & \\
\hline & -11.0000 & 2.7466 & 1.0338 & 4.0000 & 2.8793 & 0.0000 & 0.0000 & 0.0000 \\
\hline
\end{tabular}

64 ! Nr of bonds; Edis1;LPpen;n.u.;pbe1;pbo5;13corr;pbo6 pbe2;pbo3;pbo4;Etrip;pbo1;pbo2; ovcorr

$\begin{array}{rrrrrrrrrr}1 & 1 & 158.2004 & 99.1897 & 78.0000 & -0.7738 & -0.4550 & 1.0000 & 37.6117 & 0.4147 \\ & & 0.4590 & -0.1000 & 9.1628 & 1.0000 & -0.0777 & 6.7268 & 1.0000 & 0.0000 \\ & 2 & 169.4760 & 0.0000 & 0.0000 & -0.6083 & 0.0000 & 1.0000 & 6.0000 & 0.7652 \\ & & 5.2290 & 1.0000 & 0.0000 & 1.0000 & -0.0553 & 6.9316 & 0.0000 & 0.0000 \\ 2 & 2 & 153.3934 & 0.0000 & 0.0000 & -0.4600 & 0.0000 & 1.0000 & 6.0000 & 0.7300 \\ & & 6.2500 & 1.0000 & 0.0000 & 1.0000 & -0.0790 & 6.0552 & 0.0000 & 0.0000 \\ 1 & 3 & 95.6228 & 137.8227 & 68.8810 & 0.4203 & -0.3774 & 1.0000 & 18.8662 & 0.6845 \\ & & 1.1270 & -0.3355 & 9.0448 & 1.0000 & -0.1842 & 5.6293 & 0.0000 & 0.0000 \\ 3 & 3 & 142.2858 & 145.0000 & 50.8293 & 0.2506 & -0.1000 & 1.0000 & 29.7503 & 0.6051 \\ & & 0.3451 & -0.1055 & 9.0000 & 1.0000 & -0.1225 & 5.5000 & 1.0000 & 0.0000 \\ 1 & 4 & 163.8300 & 145.4458 & 89.6879 & -1.3368 & -0.3468 & 1.0000 & 27.5160 & 0.1575 \\ & & 0.1817 & -0.3114 & 7.1789 & 1.0000 & -0.2345 & 4.5111 & 1.0000 & 0.0000 \\ 3 & 4 & 116.1404 & 114.0686 & 40.0000 & 0.0754 & -0.4076 & 1.0000 & 35.0000 & 0.9157 \\ & & 0.4384 & -0.2671 & 7.0000 & 1.0000 & -0.1134 & 7.6679 & 1.0000 & 0.0000 \\ 4 & 4 & 157.9384 & 82.5526 & 152.5336 & 0.4010 & -0.1034 & 1.0000 & 12.4261 & 0.5828 \\ & & 0.1578 & -0.1509 & 11.9186 & 1.0000 & -0.0861 & 5.4271 & 1.0000 & 0.0000 \\ 2 & 3 & 160.0000 & 0.0000 & 0.0000 & -0.5725 & 0.0000 & 1.0000 & 6.0000 & 0.5626 \\ & & 1.1150 & 1.0000 & 0.0000 & 0.0000 & -0.0920 & 4.2790 & 0.0000 & 0.0000 \\ 2 & 4 & 210.1187 & 0.0000 & 0.0000 & -0.3705 & 0.0000 & 1.0000 & 6.0000 & 0.3284 \\ & & 5.8196 & 1.0000 & 0.0000 & 1.0000 & -0.1104 & 5.5184 & 0.0000 & 0.0000 \\ 1 & 5 & 150.8132 & 59.3363 & 55.2528 & -0.0628 & -0.5211 & 1.0000 & 18.9617 & 0.3219 \\ & & 0.3317 & -0.2289 & 7.5946 & 1.0000 & -0.1946 & 5.9455 & 1.0000 & 0.0000 \\ 2 & 5 & 143.4377 & 0.0000 & 0.0000 & -0.2944 & 0.0000 & 1.0000 & 6.0000 & 0.6034 \\ & & 9.5627 & 1.0000 & 0.0000 & 1.0000 & -0.0516 & 7.0960 & 1.0000 & 0.0000 \\ 3 & 5 & 0.0000 & 0.0000 & 0.0000 & 0.5563 & -0.4038 & 1.0000 & 49.5611 & 0.6000\end{array}$




\begin{tabular}{|c|c|c|c|c|c|c|c|c|c|}
\hline \multirow{3}{*}{4} & \multirow{3}{*}{5} & \multirow{2}{*}{0.4259} & \multirow{2}{*}{$\begin{array}{r}-0.4577 \\
0.0000\end{array}$} & \multirow{2}{*}{$\begin{array}{r}12.7569 \\
0.0000\end{array}$} & \multirow{2}{*}{$\begin{array}{l}1.0000 \\
0.4438\end{array}$} & \multirow{2}{*}{$\begin{array}{l}-0.1100 \\
-0.2034\end{array}$} & 7.1145 & 1.0000 & 0.0000 \\
\hline & & & & & & & 1.0000 & & \\
\hline & & & 153 & & 1.0000 & & 5.6864 & 1.0000 & 0.0000 \\
\hline \multirow[t]{2}{*}{5} & 5 & 96.1871 & 93.7006 & 68.6860 & & -0.4781 & 1.0000 & 17.8574 & 5000 \\
\hline & & 0.2723 & -0.2373 & 9.7875 & & -0.0950 & 6.4757 & & 0000 \\
\hline \multirow[t]{2}{*}{2} & 6 & 58.6896 & 0.0000 & 0.0000 & -0.0203 & -0.1418 & 1.0000 & 13.1260 & 0.0230 \\
\hline & & 8.2136 & -0.1310 & 0.0000 & 1.0000 & -0.2692 & 6.4254 & 0.0000 & 24.4461 \\
\hline 3 & 6 & 87.0227 & 0.0000 & 43.3991 & 0.0 & -0.3000 & 1.0000 & 36. & 0.0250 \\
\hline & & 0.0087 & -0.2500 & 12. & 1.0 & -0.0439 & 6.6073 & 000 & 461 \\
\hline 6 & 6 & 32.3808 & 0.0000 & 0.0000 & -0.0 & -0.2000 & 0000 & 16. & 641 \\
\hline & & 4.8726 & -0.2 & 10. & 1. & -0.0729 & 319 & 00 & 000 \\
\hline 1 & 7 & 110.0000 & 92.0000 & 0.0000 & 0.2 & -0.1418 & 1.0000 & 13. & 0.6000 \\
\hline & & 0.3601 & -0.1310 & 10.7257 & 1.0 & -0.08 & 5.3302 & & 0000 \\
\hline 2 & 7 & 0.1466 & 0.00 & 0.0000 & 0.22 & -0.1418 & 1.0000 & 13.1260 & 000 \\
\hline & & 0.3912 & -0.1 & 0. & 1.0 & -0.10 & 9.3302 & & 000 \\
\hline 3 & 7 & 201.0058 & 194.1410 & 000 & 1.6 & -0.5000 & 1.0000 & 25. & 873 \\
\hline & & 0.4358 & -0.1571 & 15. & 1. & -0.24 & 3823 & 1. & 000 \\
\hline 4 & 7 & 130.0000 & 0.00 & 0. & & -0.14 & 1.0000 & 13. & 000 \\
\hline & & 0.3601 & -0.1310 & 10.7257 & 1.0 & -0.0869 & 5.3302 & 1.0000 & 000 \\
\hline 6 & 7 & 0.1 & 0.0 & 0.0 & 0.2 & -0.50 & 000 & 35.0 & 000 \\
\hline & & 0.5 & -0. & 20. & 1. & -0. & 000 & & \\
\hline 7 & 7 & 0.0 & 0. & 0. & 0. & -0 & 000 & 35. & \\
\hline & & 0.5 & -0.5 & 20. & 1. & -0 & 000 & & \\
\hline 1 & 8 & 0.0 & 0.0 & 0. & -1 & -0.30 & 0000 & 36. & 000 \\
\hline & & 10.1 & -0.3500 & 25. & 1. & -0.10 & 8.2003 & & \\
\hline 2 & 8 & 0.0 & 0.0000 & 0. & -1. & $-0.3 c$ & 1.0000 & 36. & 00 \\
\hline & & 10.1 & -0.2 & 25. & 1. & -0 & 8.2003 & & 00 \\
\hline 3 & 8 & 45.8 & 0.0 & 0. & -0 & -0 . & 1.0000 & 36. & 105 \\
\hline & & 5.8 & -0.3 & 25. & 1 & -0. & 9140 & & 00 \\
\hline 4 & 8 & 0.0 & 0.0 & 0. & -1 & -0. & 000 & 36. & \\
\hline & & 10.1 & -0.3 & 25. & 1. & -0 & 003 & & \\
\hline 5 & 8 & 0.0 & 0.0000 & 0. & -1.0 & -0.3 & 1.0000 & 36. & 000 \\
\hline & & 10.1 & -0.3 & 25. & 1. & -0 & 2003 & & 100 \\
\hline 6 & 8 & 0.0 & 0. & 0. & 0. & -0 . & 0000 & 35. & \\
\hline & & 0.5 & -0.5 & 20. & 1. & -0 & 10. & & \\
\hline 7 & 8 & 0.0 & 0.0 & 0. & 0. & -0 & 000 & 35. & \\
\hline & & & -0.5 & 20. & & -0. & & & \\
\hline 8 & 8 & 64.4508 & 0.0000 & 0.0000 & -0.3 & 0.3000 & 0.0000 & 25. & 158 \\
\hline & & & & 12. & & & & & \\
\hline 4 & 6 & 50.0000 & 10. & 0. & -1 & -0 . & 0000 & 36. & 058 \\
\hline & & & -0 & 17. & & -0 . & & & \\
\hline 1 & 9 & 0.0000 & 0. & 0. & -0.2 & -0 . & 0000 & 36. & 82 \\
\hline & & 1.7 & -0.2 & 20. & 1. & -0 & & & 100 \\
\hline 2 & 9 & 0.0 & 00 & & -0 & -0 & 000 & 36 & \\
\hline & & 1.7 & -0. & 20. & & & & & \\
\hline 3 & 9 & 30.5 & 37. & & & & & 36 . & \\
\hline & & 0.1 & -0.2818 & 16. & & -0 . & 264 & & 00 \\
\hline 4 & 9 & 132.8785 & 36.3 & & 0. & -0 . & 000 & 36. & 50 \\
\hline & & 0.1138 & -0.2808 & 16. & 1. & -0. & 3256 & & 100 \\
\hline 5 & 9 & 106.8 & 25. & & & & & 36 & \\
\hline & & 1.3 & -0 & 15. & & -0 & & & \\
\hline 6 & 9 & 0.0 & & & -0.2 & & & 36. & \\
\hline & & & -0.2500 & 20.0000 & & & & & \\
\hline 7 & 9 & 0.0000 & 0.0000 & 0.0000 & -0.2872 & -0.3000 & 1.0000 & 36.0000 & 0.0082 \\
\hline & & & -0.2 & 20. & & & & & \\
\hline 8 & 9 & 0.1000 & 0.0000 & 0. & 0.2500 & -0.5000 & 1.0000 & 35.0000 & 6000 \\
\hline & & & -0.5000 & 20.0000 & & -0 & 10.0000 & & \\
\hline 9 & 9 & 80.1930 & 0.0000 & 0.0000 & -0.8469 & -0.2000 & 0.0000 & 16.0 & 2022 \\
\hline & & & -0.1924 & 14.9725 & & & 5.0000 & & \\
\hline 1 & 10 & 0.0000 & 0.0000 & 0.0000 & 0.5000 & -0.2000 & 0.0000 & 16.0000 & 0.5000 \\
\hline & & & & 15. & & & 10.0000 & & \\
\hline 2 & 10 & 98.9788 & 0.0000 & 0.0000 & -0.0572 & -0.2000 & 0.0000 & 16.0000 & 1.1523 \\
\hline
\end{tabular}




\begin{tabular}{|c|c|c|c|c|c|c|c|c|c|}
\hline 3 & 10 & $\begin{array}{l}2.2822 \\
0.0000 \\
1.0001\end{array}$ & $\begin{array}{r}-0.2000 \\
0.0000 \\
-0.2000\end{array}$ & $\begin{array}{r}15.0000 \\
0.0000 \\
15.0000\end{array}$ & $\begin{array}{l}1.0000 \\
0.5000 \\
1.0000\end{array}$ & $\begin{array}{l}-0.1093 \\
-0.2000 \\
-0.1000\end{array}$ & $\begin{array}{r}5.1686 \\
0.0000 \\
10.0000\end{array}$ & $\begin{array}{r}0.0000 \\
16.0000 \\
0.0000\end{array}$ & $\begin{array}{l}0.0000 \\
0.5000 \\
0.0000\end{array}$ \\
\hline 4 & 10 & $\begin{array}{l}0.0000 \\
1.0001\end{array}$ & $\begin{array}{r}0.0000 \\
-0.2000\end{array}$ & $\begin{array}{r}0.0000 \\
15.0000\end{array}$ & $\begin{array}{l}0.5000 \\
1.0000\end{array}$ & $\begin{array}{l}-0.2000 \\
-0.1000\end{array}$ & $\begin{array}{r}0.0000 \\
10.0000\end{array}$ & $\begin{array}{r}16.0000 \\
0.0000\end{array}$ & $\begin{array}{l}0.5000 \\
0.0000\end{array}$ \\
\hline 5 & 10 & 0.0000 & 0.0000 & & & & & 16. & \\
\hline 6 & 10 & $\begin{array}{l}1.0001 \\
0.0000 \\
1.0001\end{array}$ & $\begin{array}{r}-0.2000 \\
0.0000 \\
-0.2000\end{array}$ & $\begin{array}{r}15.0000 \\
0.0000 \\
15.0000\end{array}$ & $\begin{array}{l}1.0000 \\
0.5000 \\
1.0000\end{array}$ & $\begin{array}{l}-0.1000 \\
-0.2000 \\
-0.1000\end{array}$ & $\begin{array}{r}10.0000 \\
0.0000 \\
10.0000\end{array}$ & $\begin{array}{r}0.0000 \\
16.0000 \\
0.0000\end{array}$ & $\begin{array}{l}0.0000 \\
0.5000 \\
0.0000\end{array}$ \\
\hline 7 & 10 & $\begin{array}{l}0.0000 \\
1.0001\end{array}$ & $\begin{array}{r}0.0000 \\
-0.2000\end{array}$ & $\begin{array}{r}0.0000 \\
15.0000\end{array}$ & $\begin{array}{l}0.5000 \\
1.0000\end{array}$ & $\begin{array}{l}-0.2000 \\
-0.1000\end{array}$ & $\begin{array}{r}0.0000 \\
10.0000\end{array}$ & $\begin{array}{r}16.0000 \\
0.0000\end{array}$ & $\begin{array}{l}0.5000 \\
0.0000\end{array}$ \\
\hline 8 & 10 & $\begin{array}{l}0.0000 \\
1.0001\end{array}$ & $\begin{array}{r}0.0000 \\
-0.2000\end{array}$ & $\begin{array}{r}0.0000 \\
15.0000\end{array}$ & & $\begin{array}{l}-0.2000 \\
-0.1000\end{array}$ & $\begin{array}{r}0.0000 \\
10.0000\end{array}$ & $\begin{array}{r}16.0000 \\
0.0000\end{array}$ & $\begin{array}{l}0.5000 \\
0.0000\end{array}$ \\
\hline 9 & 10 & $\begin{array}{l}0.0000 \\
1.0001\end{array}$ & $\begin{array}{r}0.0000 \\
-0.2000\end{array}$ & $\begin{array}{r}0 . \\
15 .\end{array}$ & & $\begin{array}{l}-0.2000 \\
-0.1000\end{array}$ & & $\begin{array}{r}16 \\
0\end{array}$ & \\
\hline 0 & 10 & $\begin{array}{l}0.2500 \\
0.9228\end{array}$ & $\begin{array}{r}0.0000 \\
-0.2000\end{array}$ & & $\begin{array}{l}0.1 \\
1.0\end{array}$ & $\begin{array}{l}-0.2000 \\
-0.1178\end{array}$ & & & $\begin{array}{l}3356 \\
0000\end{array}$ \\
\hline 1 & 11 & $\begin{array}{r}237.8781 \\
3.6661\end{array}$ & $\begin{array}{r}0.0000 \\
-0.2500\end{array}$ & $\begin{array}{r}0.0000 \\
15.0000\end{array}$ & $\begin{array}{r}-0.7438 \\
1.0000\end{array}$ & $\begin{array}{l}-0.5000 \\
-0.0800\end{array}$ & & $\begin{array}{r}35.0000 \\
1.0000\end{array}$ & \\
\hline 2 & 11 & $\begin{array}{r}0.0000 \\
12.3710\end{array}$ & $\begin{array}{l}0.00 \\
1.00\end{array}$ & & $\begin{array}{r}-0.4 \\
1.0\end{array}$ & $\begin{array}{r}0.0000 \\
-0.1008\end{array}$ & & & \\
\hline 3 & 11 & $\begin{array}{r}0.0000 \\
12.3710\end{array}$ & & & 43 & $\begin{array}{r}0.0000 \\
-0.1008\end{array}$ & & & \\
\hline 4 & 11 & $\begin{array}{r}0.0000 \\
12.3710\end{array}$ & $\begin{array}{l}0.00 \\
1.00\end{array}$ & 0.1 & $\begin{array}{r}-0.4 \\
1.0\end{array}$ & $\begin{array}{r}0.00 \\
-0.10\end{array}$ & & & $\begin{array}{l}6151 \\
0000\end{array}$ \\
\hline 5 & 11 & 0.0000 & 0.0 & 0. & -0.4 & 0.0000 & 000 & 000 & 6151 \\
\hline & 11 & $\begin{array}{r}12.3710 \\
250.0765\end{array}$ & & & & $\begin{array}{l}-0.1008 \\
-0.3500\end{array}$ & & & $\begin{array}{l}0000 \\
8427\end{array}$ \\
\hline & & 0.1167 & -0.2500 & 15. & 1.0000 & -0.1506 & 516 & 000 & 0.0000 \\
\hline 1 & 12 & $\begin{array}{r}85.5942 \\
0.1726\end{array}$ & $\begin{array}{r}0.0000 \\
-0.2000\end{array}$ & $\begin{array}{r}0.6 \\
15.0\end{array}$ & $\begin{array}{l}0.3 \\
1.0\end{array}$ & $\begin{array}{l}-0.2 \\
-0.1\end{array}$ & $\begin{array}{l}000 \\
607\end{array}$ & $\begin{array}{r}16 . \\
0 .\end{array}$ & $\begin{array}{l}0.1488 \\
0.0000\end{array}$ \\
\hline 2 & 12 & $\begin{array}{r}121.8652 \\
0.0683\end{array}$ & $\begin{array}{r}0.0000 \\
-0.2000\end{array}$ & $\begin{array}{r}0 . \mathrm{C} \\
15 . \mathrm{C}\end{array}$ & $\begin{array}{l}0.3 \\
1.0\end{array}$ & $\begin{array}{l}-0.2 \\
-0.0\end{array}$ & $\begin{array}{l}000 \\
834\end{array}$ & $\begin{array}{l}000 \\
000\end{array}$ & $\begin{array}{l}0.2297 \\
0.0000\end{array}$ \\
\hline 3 & 12 & $\begin{array}{r}110.9688 \\
0.1356\end{array}$ & $\begin{array}{r}0.0000 \\
-0.2000\end{array}$ & $\begin{array}{r}0.0000 \\
15.0000\end{array}$ & $\begin{array}{r}-0.0011 \\
1.0000\end{array}$ & $\begin{array}{l}-0.2000 \\
-0.1972\end{array}$ & $\begin{array}{l}1.0000 \\
6.1209\end{array}$ & $\begin{array}{r}16.0000 \\
1.0000\end{array}$ & $\begin{array}{l}0.1488 \\
0.0000\end{array}$ \\
\hline 4 & 12 & $\begin{array}{r}116.7085 \\
0.8641\end{array}$ & $\begin{array}{r}0.0000 \\
-0.2000\end{array}$ & $\begin{array}{r}0.0000 \\
15.0000\end{array}$ & $\begin{array}{r}-0.9802 \\
1.0000\end{array}$ & $\begin{array}{r}0.0000 \\
-0.0510\end{array}$ & $\begin{array}{l}1.0000 \\
8.4754\end{array}$ & $\begin{array}{r}16.0000 \\
0.0000\end{array}$ & $\begin{array}{l}0.8958 \\
0.0000\end{array}$ \\
\hline 5 & 12 & $\begin{array}{r}142.5975 \\
-0.7617\end{array}$ & $\begin{array}{r}0.0000 \\
-0.2000\end{array}$ & $\begin{array}{r}0.0000 \\
15.0000\end{array}$ & $\begin{array}{l}0.3974 \\
1.0000\end{array}$ & $\begin{array}{l}-0.2000 \\
-0.0957\end{array}$ & $\begin{array}{l}0.0000 \\
5.5450\end{array}$ & $\begin{array}{r}16.0000 \\
0.0000\end{array}$ & $\begin{array}{l}0.1800 \\
0.0000\end{array}$ \\
\hline & 12 & $\begin{array}{r}146.6542 \\
0.2793\end{array}$ & $\begin{array}{r}0.0000 \\
-0.2000\end{array}$ & $\begin{array}{r}0.0000 \\
15.0000\end{array}$ & $\begin{array}{r}-0.0295 \\
1.0000\end{array}$ & $\begin{array}{l}-0.2000 \\
-0.1591\end{array}$ & $\begin{array}{l}0.0000 \\
5.3892\end{array}$ & $\begin{array}{r}16.0000 \\
0.0000\end{array}$ & $\begin{array}{l}0.3319 \\
0.0000\end{array}$ \\
\hline
\end{tabular}

29 ! $\mathrm{Nr}$ of off-diagonal terms; $\mathrm{E}$

(1)

$\begin{array}{rrrrrrrr}1 & 2 & 0.1239 & 1.4004 & 9.8467 & 1.1210 & -1.0000 & -1.0000 \\ 2 & 3 & 0.0283 & 1.2885 & 10.9190 & 0.9215 & -1.0000 & -1.0000 \\ 2 & 4 & 0.1294 & 1.3025 & 9.8751 & 1.0415 & -1.0000 & -1.0000 \\ 1 & 3 & 0.0628 & 1.9606 & 10.0282 & 1.3240 & 1.2084 & 1.0739 \\ 1 & 4 & 0.2000 & 1.8828 & 9.7673 & 1.3387 & 1.2578 & 1.1539 \\ 3 & 4 & 0.1001 & 2.3274 & 9.0974 & 1.3745 & 1.0561 & 1.0871 \\ 1 & 5 & 0.1408 & 1.8161 & 9.9393 & 1.7986 & 1.3021 & 1.4031 \\ 2 & 5 & 0.0895 & 1.6239 & 10.0104 & 1.4640 & -1.0000 & -1.0000 \\ 3 & 5 & 0.1022 & 1.9887 & 10.0605 & 1.5799 & 1.4000 & -1.0000 \\ 4 & 5 & 0.1505 & 1.9000 & 10.5104 & 1.8000 & 1.4000 & -1.0000 \\ 2 & 6 & 0.0100 & 1.6000 & 13.2979 & 1.8670 & -1.0000 & -1.0000 \\ 3 & 6 & 0.0809 & 1.7000 & 11.4606 & 1.5177 & -1.0000 & -1.0000 \\ 3 & 7 & 0.0534 & 1.7520 & 10.4281 & 1.8000 & 1.4498 & -1.0000 \\ 6 & 7 & 0.1801 & 1.8566 & 9.8498 & 0.1000 & -1.0000 & -1.0000 \\ 3 & 8 & 0.0825 & 1.5904 & 11.3396 & 1.5905 & -1.0000 & -1.0000 \\ 2 & 9 & 0.1750 & 1.7939 & 13.5000 & 0.0100 & -1.0000 & -1.0000 \\ 3 & 9 & 0.1200 & 1.8000 & 10.5000 & 1.6526 & 1.4718 & -1.0000 \\ 1 & 9 & 0.2950 & 2.2000 & 11.0937 & 0.0100 & -1.0000 & -1.0000 \\ 2 & 10 & 0.0376 & 1.6671 & 9.6285 & 1.2123 & -1.0000 & -1.0000 \\ 3 & 10 & 0.1945 & 2.2766 & 11.2353 & -1.0000 & -1.0000 & -1.0000\end{array}$




\begin{tabular}{|c|c|c|c|c|c|c|c|c|c|c|}
\hline 1 & 11 & \multicolumn{2}{|c|}{0.1071} & 1.6243 & \multicolumn{2}{|c|}{11.0402} & 1.3176 & -1.0000 & -1.0000 & \\
\hline 2 & 11 & \multicolumn{2}{|c|}{0.0431} & 1.7204 & \multicolumn{2}{|c|}{10.3632} & 0.5386 & -1.0000 & -1.0000 & \\
\hline 1 & 12 & \multirow{2}{*}{\multicolumn{2}{|c|}{0.1910}} & 2.0244 & & 6929 & 1.8040 & -1.0000 & -1.0000 & \\
\hline 2 & 12 & & & 1.6944 & & 9802 & 1.3305 & -1.0000 & -1.0000 & \\
\hline 3 & 12 & & .1044 & 2.3847 & & 6146 & 2.0355 & -1.0000 & -1.0000 & \\
\hline 4 & 12 & & .8000 & 1.1534 & & 3829 & 1.3945 & -1.0000 & -1.0000 & \\
\hline 5 & 12 & & .2257 & 2.2125 & & 2051 & 2.1505 & -1.0000 & -1.0000 & \\
\hline 4 & 9 & & .1254 & 1.8020 & & 5000 & 1.6560 & 1.4551 & -1.0000 & \\
\hline 5 & 9 & & .2224 & 2.0110 & & 360 & 2.2025 & 1.6765 & -1.0000 & \\
\hline 29 & & ! & $r$ of ang & les; at & - at & ; at3; Th & netao, o; ka & $a ; k b ; p v 1 ; p$ & pv2 & \\
\hline 1 & 1 & 1 & 59.0573 & 30.70 & & 0.7606 & 0.0000 & $0 \quad 0.7180$ & 6.2933 & 1.1244 \\
\hline 1 & 1 & 2 & 65.7758 & 14.5 & & 6.2481 & 0.0000 & 0.5665 & 0.0000 & 1.6255 \\
\hline 2 & 1 & 2 & 70.2607 & 25.22 & & 3.7312 & 0.0000 & 0.0050 & 0.0000 & 2.7500 \\
\hline 1 & 2 & 2 & 0.0000 & 0.0 & & 6.0000 & 0.0000 & 0.0000 & 0.0000 & 1.0400 \\
\hline 1 & 2 & 1 & 0.0000 & 3.41 & & 7.7350 & 0.0000 & 0.0000 & 0.0000 & 1.0400 \\
\hline 2 & 2 & 2 & 0.0000 & 27.92 & & 5.8635 & 0.0000 & 0.0000 & 0.0000 & 1.0400 \\
\hline 1 & 1 & 3 & 68.3591 & 25.83 & & 1.4410 & 0.0000 & 2.9950 & 58.6562 & 0207 \\
\hline 3 & 1 & 3 & 86.6618 & 25.13 & & 5.1177 & -1.0000 & 1.5183 & 0.0000 & 2.9671 \\
\hline 1 & 1 & 4 & 64.7353 & 38.2 & & 1.1478 & 0.0000 & 1.1834 & 0.0000 & 2.8465 \\
\hline 3 & 1 & 4 & 81.0672 & 41.9 & & 0.4878 & 0.0000 & 1.1019 & 0.0000 & 1.0000 \\
\hline 4 & 1 & 4 & 89.7621 & 43.0 & & 0.5895 & 0.0000 & 1.1155 & 0.0000 & 1.0000 \\
\hline 2 & 1 & 3 & 63.0181 & 30.45 & & 2.5496 & 0.0000 & 0.1000 & 0.0000 & 1.2924 \\
\hline 2 & 1 & 4 & 68.7361 & 36.71 & & 1.6697 & 0.0000 & 0.2000 & 0.0000 & 000 \\
\hline 1 & 2 & 4 & 0.0000 & 0.0 & & 6.3000 & 0.0000 & 0.0000 & 0.0000 & 1.0400 \\
\hline 1 & 3 & 1 & 68.5087 & 45.0 & & 1.4583 & 0.0000 & 2.8294 & 0.0000 & 1.0000 \\
\hline 1 & 3 & 3 & 80.5221 & 44.23 & & 1.5331 & 0.0000 & 1.0572 & 68.1072 & 1.3414 \\
\hline 1 & 3 & 4 & 70.3730 & 45.0 & & 1.4731 & 0.0000 & 2.9000 & 0.0000 & 2.4464 \\
\hline 3 & 3 & 3 & 89.9974 & 18.21 & & 1.8037 & 0.0000 & 2.9881 & 0.0000 & 1.0615 \\
\hline 3 & 3 & 4 & 77.0669 & 27.67 & & 1.6466 & 0.0000 & 2.9000 & 0.0000 & 085 \\
\hline 4 & 3 & 4 & 68.8583 & 40.47 & & 1.8369 & 0.0000 & 3.0072 & 0.0000 & 1.5773 \\
\hline 1 & 3 & 2 & 90.0000 & 7.28 & & 7.5000 & 0.0000 & 1. 3111 & 0.0000 & 3.0000 \\
\hline 2 & 3 & 3 & 75.6935 & 50.0 & & 2.0000 & 0.0000 & 1.0000 & 0.0000 & 680 \\
\hline 2 & 3 & 4 & 68.3253 & 36.1 & & 7.5000 & 0.0000 & 0.1000 & 0.0000 & 1.0000 \\
\hline 2 & 3 & 2 & 85.8000 & 9.8 & & 2.2720 & 0.0000 & 2.8635 & 0.0000 & 1.5800 \\
\hline 1 & 4 & 1 & 71.2077 & 14.1 & & 3. 3944 & 0.0000 & 2.8702 & 0.0000 & 1. 2651 \\
\hline 1 & 4 & 3 & 76.1064 & 23.7 & & 1.6308 & 0.0000 & 2.8701 & 0.00 & 732 \\
\hline 1 & 4 & 4 & 71.3624 & 12.8 & & 3. 1458 & 0.0000 & 2.8701 & 0.0000 & 1.1896 \\
\hline 3 & 4 & 3 & 74.2922 & 23.0 & & 2.6248 & -18.0069 & 3.0701 & 0.0000 & 1.6278 \\
\hline 3 & 4 & 4 & 74.0840 & 31.1 & & 1.5175 & -0.9193 & 3.0117 & 0.0000 & 1.3541 \\
\hline 4 & 4 & 4 & 76.0945 & 32.1 & & 1.7767 & 0.0000 & 2.9983 & 0.0000 & 1.9677 \\
\hline 1 & 4 & 2 & 69.1892 & 14.8 & & 2.7174 & 0.0000 & 0.2025 & $0.0 C$ & 071 \\
\hline 2 & 4 & 3 & 74.5555 & 45.0 & & 1. 1948 & 0.0000 & 0.3956 & 0.0000 & 000 \\
\hline 2 & 4 & 4 & 78.8758 & 45.0 & & 0.5964 & 0.0000 & 0.5437 & 0.0000 & 1.0000 \\
\hline 2 & 4 & 2 & 81.5738 & 7.0 & & 7.5000 & 0.0000 & 0.1000 & 0.0000 & 1.0000 \\
\hline 1 & 2 & 3 & 0.0000 & 13.2 & & 1.9388 & 0.0000 & 0.0000 & 0.0000 & 1.0000 \\
\hline 1 & 2 & 4 & 0.0000 & 0.0 & & 2.4974 & 0.0000 & 0.0000 & 0.0000 & 1.3777 \\
\hline 1 & 2 & 5 & 0.0000 & 15.0 & & 3.0000 & 0.0000 & 0.0000 & 0.0000 & 1.0400 \\
\hline 3 & 2 & 3 & 0.0000 & 15.0 & & 2.8900 & 0.0000 & 0.0000 & 0.0000 & 2.8774 \\
\hline 3 & 2 & 4 & 0.0000 & 1.0 & & 0.1000 & 0.0000 & 0.0000 & 0.0000 & 3.0000 \\
\hline 4 & 2 & 4 & 0.0000 & 0.0 & & 1. 3170 & 0.0000 & 0.0000 & 0.0000 & 2.1165 \\
\hline 2 & 2 & 3 & 0.0000 & 8.5 & & 3.0000 & 0.0000 & 0.0000 & 0.0000 & 1.0421 \\
\hline 2 & 2 & 4 & 0.0000 & 0.0 & & 6.0000 & 0.0000 & 0.0000 & 0.0000 & 1.0400 \\
\hline 1 & 1 & 5 & 74.4180 & 33.4 & & 1.7018 & 0.1463 & 0.5000 & 0.0000 & 1.6178 \\
\hline 1 & 5 & 1 & 79.7037 & 28.2 & & 1.7073 & 0.1463 & 0.5000 & 0.0000 & 1.6453 \\
\hline 2 & 1 & 5 & 63.3289 & 29.4 & & 2.1326 & 0.0000 & 0.5000 & 0.0000 & 3.0000 \\
\hline 1 & 5 & 2 & 85.9449 & 38.3 & & 1.2492 & 0.0000 & 0.5000 & 0.0000 & 1.1000 \\
\hline 1 & 5 & 5 & 85.6645 & 40.0 & & 2.9274 & 0.1463 & 0.5000 & 0.0000 & 1.3830 \\
\hline 2 & 5 & 2 & 83.8555 & 5.1 & & 0.4377 & 0.0000 & 0.5000 & 0.0000 & 3.0000 \\
\hline 2 & 5 & 5 & 97.0064 & 32.1 & & 2.0242 & 0.0000 & 0.5000 & 0.0000 & 2.8568 \\
\hline 3 & 5 & 3 & 81.0926 & 30.2 & & 6.4132 & -5.4471 & 2.5968 & 0.0000 & 3.0000 \\
\hline 1 & 5 & 3 & 70.0000 & 35.0 & & 3.4223 & 0.0000 & 1.3550 & 0.0000 & 1.2002 \\
\hline 1 & 3 & 5 & 57.3353 & 41.0 & & 1.0609 & 0.0000 & 1.3000 & 0.0000 & 3.0000 \\
\hline
\end{tabular}




\begin{tabular}{|c|c|c|c|c|c|c|c|c|c|}
\hline 3 & 3 & 5 & 83.9753 & 31.0715 & 3.5590 & 0.0000 & 0.8161 & 0.0000 & 1.1776 \\
\hline 2 & 3 & 5 & 89.8843 & 17.5000 & .3660 & 0.0000 & 2.0000 & 0.0000 & 2.0734 \\
\hline 2 & 6 & 2 & 0.0000 & 49.8261 & 0.2093 & 0.0000 & 2.0870 & 0.0000 & 2.2895 \\
\hline 2 & 2 & 6 & 0.0000 & 40.0366 & .1505 & 0.0000 & 1.1296 & 0.0000 & 1.1110 \\
\hline 6 & 2 & 6 & 0.0000 & 0.5047 & .8000 & 0.0000 & 0.8933 & 0.0000 & 4.6650 \\
\hline 2 & 6 & 6 & 0.0000 & 8.7037 & .0827 & 0.0000 & 3.5597 & 0.0000 & 1.1198 \\
\hline 3 & 6 & 3 & 0.0000 & 9.2317 & 0.1000 & 0.0000 & 1.0000 & 0.0000 & 1.0920 \\
\hline 6 & 3 & 6 & 0.0008 & 25.0000 & 8.0000 & 0.0000 & 1.0000 & 0.0000 & 3.0000 \\
\hline 2 & 3 & 6 & 66.0423 & 5.0000 & 1.0000 & 0.0000 & 1.0000 & 0.0000 & 1.2500 \\
\hline 2 & 6 & 3 & 0.0000 & 0.5000 & .1000 & 0.0000 & 1.0000 & 0.0000 & 3.0000 \\
\hline 3 & 3 & 6 & 70.0000 & 20.0000 & 1.0000 & 0.0000 & 1.0000 & 0.0000 & 1.2500 \\
\hline 3 & 7 & 3 & 90.0000 & 18.4167 & 0.6799 & -8.0000 & 0.1310 & 0.0000 & 2.2321 \\
\hline 2 & 3 & 7 & 72.6004 & 9.6150 & 0.8905 & 0.0000 & 3.5473 & 0.0000 & 1.0400 \\
\hline 3 & 3 & 7 & 60.0000 & 40.0000 & 4.0000 & 0.0000 & 1.0000 & 0.0000 & 1.0400 \\
\hline 3 & 2 & 7 & 0.0000 & 10.0000 & 1.0000 & 0.0000 & 1.0000 & 0.0000 & 1.0400 \\
\hline 6 & 3 & 7 & 41.0995 & 3.2207 & 7.3523 & 0.0000 & 0.1101 & 0.0000 & 1.0947 \\
\hline 7 & 3 & 7 & 62.1312 & 7.5931 & 0.1000 & 0.0000 & 0.5154 & 0.0000 & 2.1744 \\
\hline 1 & 3 & 7 & 74.1 & 8.5687 & 1.7132 & 0.0000 & -0.6553 & 0.0000 & 2.2323 \\
\hline 2 & 7 & 3 & 75.0000 & 25.0000 & 2.0000 & 0.0000 & 1.0000 & 0.0000 & 1.2500 \\
\hline 3 & 7 & 7 & 70.0000 & 25.0000 & 2.0000 & 0.0000 & 1.0000 & 0.0000 & 1.2500 \\
\hline 3 & 9 & 3 & 90.0 & 30.46 & 468 & 0.0000 & 0.0500 & 0.0000 & 1.9485 \\
\hline 9 & 3 & 9 & 90.0 & 5.7 & 000 & 0.0000 & 2.0000 & 0.0000 & 1.1000 \\
\hline 3 & 3 & 9 & 62.5 & 15. & 743 & 000 & & 0.0000 & 1.1673 \\
\hline 3 & 9 & 9 & 33.7 & & & 000 & & 0.0000 & 2.6065 \\
\hline 2 & 3 & 9 & 90. & & & 000 & & 0.0000 & 1.7257 \\
\hline 1 & 3 & 9 & 90.0 & 11. & & 000 & & 0.0000 & 2.1105 \\
\hline 3 & 2 & 10 & 0.0 & & & & & & 1456 \\
\hline 11 & 11 & 11 & 77.8443 & $49 . c$ & 913 & 000 & 0.7835 & 0.0000 & 2.3020 \\
\hline 1 & 11 & 1 & 0.0 & 19. & & 000 & & 0.0000 & 1.1537 \\
\hline 1 & 11 & 11 & 0.0 & 25. & & 000 & & 0.0000 & 1.0400 \\
\hline 11 & 1 & 2 & 69.6 & 10. & 00 & 000 & & 0.0000 & 1.0400 \\
\hline 4 & 9 & 4 & 90.0 & 30. & & 000 & & 0.0000 & 1.9485 \\
\hline 3 & 9 & 4 & 90.0 & 30. & & 000 & 0.1 & 0.0000 & 1.9485 \\
\hline 9 & 4 & 9 & 90. & & & & & 0.0000 & 1.1000 \\
\hline 3 & 4 & 9 & 62.5 & 15. & & 000 & & 0.0000 & 1.1673 \\
\hline 4 & 3 & 9 & 62.5 & & & & & 0.0000 & \\
\hline 4 & 4 & 9 & 62.9 & 15. & & 900 & & 0.0000 & 1.1673 \\
\hline 4 & 4 & 9 & 62.9 & & & & & 0.0000 & 1673 \\
\hline 4 & 9 & 9 & 33.7 & & & & & 0.0000 & 2.6065 \\
\hline 2 & 4 & 9 & 90.0 & & & & & 0.0000 & 1.7257 \\
\hline 1 & 4 & 9 & 8 & 10. & & & & & \\
\hline 3 & 9 & 5 & $90 . \mathrm{C}$ & 10. & & 000 & & 0.0000 & 2.0000 \\
\hline 4 & 9 & 5 & 90. & 30. & & & & & \\
\hline 5 & 9 & 5 & 90. & 30. & & 000 & & 0.0 & 485 \\
\hline 5 & 9 & 9 & 33. & & & & & & \\
\hline 9 & 5 & 9 & 90. & & & & & 0.0 & 000 \\
\hline 3 & 5 & 9 & 62. & 15. & & & & 0.0000 & 1.1673 \\
\hline 5 & 3 & 9 & & & & & & & 1.1673 \\
\hline 4 & 5 & 9 & 62.9 & 15. & & & & 0.0000 & 1.1673 \\
\hline 5 & 4 & 9 & & & & & & & \\
\hline 5 & 5 & 9 & & 15. & & & & & 1.1673 \\
\hline 2 & 5 & 9 & & & & & & & \\
\hline 1 & 5 & 9 & & & & & & & 1.3611 \\
\hline 1 & 1 & 12 & & & & & & & \\
\hline 2 & 1 & 12 & & & & & & & 2.5627 \\
\hline 5 & & 12 & & & & & & & \\
\hline 1 & 5 & 12 & & & & & & & 1.1255 \\
\hline 2 & 5 & 12 & 82.2 & 23. & & & & & 2.4404 \\
\hline 5 & 5 & 12 & & & & & & & \\
\hline & 12 & 1 & 71.3 & 3.9 & 8 & 000 & 1.1 & 0.0000 & 2.1341 \\
\hline & 12 & 2 & 63.1 & & & & & & \\
\hline & 12 & 5 & 33.9374 & 50.0000 & 1.6182 & 0.0000 & 0.1948 & 0.0000 & 2.5397 \\
\hline
\end{tabular}




\begin{tabular}{|c|c|c|c|c|c|c|c|c|c|c|}
\hline 2 & 12 & 2 & \multicolumn{2}{|c|}{47.1487} & 9.2600 & 2.9275 & 0.0000 & 1.3836 & 0.0000 & 2.5657 \\
\hline 2 & 12 & 5 & \multicolumn{2}{|c|}{56.6111} & 27.1999 & 3.8869 & 0.0000 & 0.9903 & 0.0000 & 1.4852 \\
\hline 5 & 12 & 5 & & .0000 & 24.0506 & 2.1768 & 000 & 0.5636 & 0.0000 & 1.7970 \\
\hline 1 & 12 & 12 & & .0000 & 7.2189 & 1.6647 & 0.0000 & 0.9966 & 0.0000 & 1.2857 \\
\hline 2 & 12 & 12 & & .9056 & 9.2673 & 0.1644 & 0000 & 1.0082 & 0.0000 & 1.0000 \\
\hline 5 & 12 & 12 & & .2439 & 17.8425 & 2.8942 & 0.0000 & 1.0982 & 0.0000 & 2.3376 \\
\hline 12 & 5 & 12 & & .1787 & 11.1483 & 3.6845 & 000 & 0.5612 & 0.0000 & 1.5584 \\
\hline 2 & 3 & 12 & & .0000 & 10.0000 & 1.0000 & 0.0000 & 1.0500 & 0.0000 & 1.2500 \\
\hline 3 & 2 & 12 & & .0000 & 5.0000 & 2.0000 & 0.0000 & 1.0000 & 0.0000 & 500 \\
\hline 2 & 12 & 3 & & .0000 & 10.0000 & 2.0000 & 0.0000 & 1.0000 & 0.0000 & 1.2500 \\
\hline 3 & 3 & 12 & & . 3915 & 36.3302 & 1.5083 & 0.0000 & 1.0000 & 0.0000 & 1.5506 \\
\hline 3 & 12 & 3 & & .1302 & 7.6175 & 6.2730 & 0.0000 & 0.5000 & 9000 & 1.0917 \\
\hline 12 & 3 & 12 & & .1000 & 2.8512 & 8.0000 & 0.00 & 0.5000 & 0.0000 & 1.0000 \\
\hline 3 & 12 & 12 & & .7044 & 15.5303 & 0.5696 & 0.0000 & 0.1000 & 0.0000 & 1.5 \\
\hline 1 & 4 & 12 & 106 & .6991 & 17.7341 & 3.6201 & 0.0000 & 0.9716 & 0.0000 & 1.1 \\
\hline 9 & & & & $f$ torsio & ons; at 1 ; at & $-2.0+2.0+1$ & $4 ; ; \mathrm{V} 1 ; \mathrm{V} 2 ; \mathrm{V}$ & 13; V2(B0); & onj; n.u; & \\
\hline 1 & 1 & 1 & 1 & -0.2500 & 34.7453 & 0.0288 & -6.3507 & -1.6000 & 0.0000 & 0.0000 \\
\hline 1 & 1 & 1 & 2 & -0.2500 & 29.2131 & 0.2945 & -4.9581 & -2.1802 & 0.0000 & .0000 \\
\hline 2 & 1 & 1 & 2 & -0.2500 & 31.2081 & 0.4539 & -4.8923 & -2.2677 & 0.0000 & .0000 \\
\hline 1 & 1 & 1 & 3 & 0.5784 & 25.7360 & 0.1495 & -6.0432 & -1.1000 & 0.0000 & 0.0000 \\
\hline 2 & 1 & 1 & 3 & 1.8866 & 44.6425 & 0.9109 & -7.5698 & -1.0978 & 0.0000 & .0000 \\
\hline 3 & 1 & 1 & 3 & -2.5000 & 28.3931 & -1.0000 & -2.5006 & -0.8614 & 0.00 & .0000 \\
\hline 1 & 1 & 3 & 1 & 2.3970 & 26.6083 & 1.0000 & -3.3221 & -0.9000 & 0.0000 & 0.0000 \\
\hline 1 & 1 & 3 & 2 & -2.5000 & 9.7539 & -1.0000 & -2.5000 & -0.9000 & 0.0000 & .0000 \\
\hline 2 & 1 & 3 & 1 & -1.6106 & 32.4873 & 0.6315 & -2.7195 & -0.9000 & 0.00 & .0000 \\
\hline 2 & 1 & 3 & 2 & -1.0000 & 53.3434 & 0.6937 & -4.8807 & -1.1000 & 0.0000 & 0.0000 \\
\hline 1 & 1 & 3 & 3 & 1.2668 & 7.0865 & 0.1813 & -5.1135 & -2.0544 & 0.00 & .0000 \\
\hline 2 & 1 & 3 & 3 & 2.5000 & 54.517 & 1.000 & -2.5 & -2.8274 & 0 & 00 \\
\hline 3 & 1 & 3 & 1 & 1.1496 & 80.0000 & -0.0611 & -4.5335 & -3.0437 & 0.00 & .0000 \\
\hline 3 & 1 & 3 & 2 & -1.0000 & 90.6930 & -0.4162 & .8029 & .0476 & .00 & .0000 \\
\hline 3 & 1 & 3 & 3 & -0.7819 & 24.1545 & 0.6237 & -4.0590 & -2.7174 & .00 & .0000 \\
\hline 1 & 3 & 3 & 1 & -0.7439 & 1.6939 & 0.9407 & -2.5051 & -2.7536 & .00 & .0000 \\
\hline 1 & 3 & 3 & 2 & -2.5000 & -1.8552 & 0.0789 & -5.2078 & -2.9498 & 0.0000 & .0000 \\
\hline 2 & 3 & 3 & 2 & -1.6122 & -25.0000 & -1.00 & -2.53 & -0.99 & 0 & .0000 \\
\hline 1 & 3 & 3 & 3 & 2.5000 & -22.1016 & 0.9647 & -2.6000 & -0.9972 & 0.00 & 0000 \\
\hline 2 & 3 & 3 & 3 & -2.5000 & 75.7606 & -0.61 & -7.863 & .2407 & 0 & 0000 \\
\hline 3 & 3 & 3 & 3 & -2.5000 & -25.0000 & 1.0000 & -2.5000 & -0.9000 & .00 & 0000 \\
\hline 1 & 1 & 4 & 2 & 0.2700 & 43.1323 & -0.4952 & -7.6538 & -1.9825 & 0.00 & 0000 \\
\hline 2 & 1 & 4 & 2 & -0.5047 & 82.9784 & 0.8701 & -7.6680 & -2.1051 & 0.0000 & .0000 \\
\hline 3 & 1 & 4 & 2 & 0.8306 & 15.530 & 1.00 & -2.5000 & -2.5261 & 0.00 & .0000 \\
\hline 3 & 1 & 1 & 4 & -0.8051 & 19.83 & 1.00 & -3 & -0 & & \\
\hline 4 & 1 & 1 & 4 & 1.0000 & 36.1913 & 1.0000 & -3.4095 & -1.7241 & 00 & .0000 \\
\hline 1 & 1 & 4 & 1 & 1.0000 & 32.6616 & 0.348 & -6.4524 & -1.6589 & 0.00 & 0000 \\
\hline 3 & 1 & 4 & 1 & -1.0000 & -5.000 & 1.000 & -2.5000 & -1.8038 & .06 & 0000 \\
\hline 2 & 1 & 1 & 4 & 0.7529 & 50.801 & -0.500 & -4.3471 & -1.9000 & 0.00 & .0000 \\
\hline 4 & 1 & 4 & 2 & 0.3787 & 13.73 & 0.65 & -8.25 & -2.0202 & & 0000 \\
\hline 2 & 1 & 4 & 1 & -1.0000 & 76.718 & 0.11 & -8.0000 & -1.5996 & 0 & 0000 \\
\hline 0 & 1 & 2 & 0 & 0.0000 & 0.00 & 0.00 & 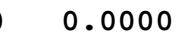 & 000 & & .0000 \\
\hline 0 & 2 & 2 & 0 & 0.0000 & 0.0000 & 0.0000 & 0.0000 & 0.0000 & 0.00 & .0000 \\
\hline 0 & 2 & 3 & 0 & 0.0000 & 0.100 & 0.02 & -2.5415 & 0.0000 & 0.00 & 0000 \\
\hline 0 & 1 & 1 & 0 & 0.0000 & 50.000 & 0.30 & -4.0000 & -2.0000 & 0.00 & 0000 \\
\hline 0 & 3 & 3 & 0 & 0.5511 & 25.415 & 1.1 & -5.19 & -1.0000 & 0 & 9000 \\
\hline 0 & 1 & 4 & 0 & 0.3636 & 41.4126 & 0.6095 & -3.7375 & -2.0051 & 0.0000 & .0000 \\
\hline 0 & 2 & 4 & 0 & 0.0000 & 0.1032 & 0.3000 & -5.0965 & 0.0000 & 0.0000 & 0.0000 \\
\hline 0 & 3 & 4 & 0 & 1.0000 & 60.0000 & 0.5000 & -3.5000 & -0.2500 & 0.0000 & .0000 \\
\hline 0 & 4 & 4 & 0 & 0.7265 & 44.3155 & 1.0000 & -4.4046 & -2.0000 & 0.0000 & 0.0000 \\
\hline 4 & 1 & 4 & 4 & -0.0949 & 8.7582 & 0.3310 & -7.9430 & -2.0000 & 0.0000 & .0000 \\
\hline 0 & 1 & 5 & 0 & 0.7575 & 50.7519 & 1.015 & -3.5255 & 0.0000 & 0.0000 & 0.0000 \\
\hline 0 & 5 & 5 & 0 & -0.0170 & 5.0786 & 0.613 & -5.0002 & 0.0000 & 0.0000 & 0.0000 \\
\hline 0 & 2 & 5 & 0 & 0.0000 & 0.0000 & 0.0000 & 0.0000 & 0.0000 & 0.0000 & 0.0000 \\
\hline 2 & 3 & 5 & 3 & 2.5000 & 2.5000 & 0.2237 & -10.0000 & -1.0000 & 0.0000 & 0.0000 \\
\hline 0 & 3 & 5 & 0 & -2.5000 & 50.0000 & -0.5000 & -10.0000 & -1.0000 & 0.0000 & 0.0000 \\
\hline
\end{tabular}




\begin{tabular}{|c|c|c|c|c|c|c|c|c|c|c|c|}
\hline 0 & 6 & 6 & 0 & \multicolumn{2}{|c|}{0.0000} & 0.0000 & 0.1200 & -2.4426 & 0.0000 & 0.0000 & 0.0000 \\
\hline 0 & 2 & 6 & 0 & \multicolumn{2}{|c|}{0.0000} & 0.0000 & 0.1200 & -2.4847 & 0.0000 & 0.0000 & 0.0000 \\
\hline 0 & 3 & 6 & 0 & \multicolumn{2}{|c|}{0.0000} & 0.0000 & 0.1200 & -2.4703 & 0.0000 & 0.0000 & 0.0000 \\
\hline 1 & 1 & 3 & 3 & \multicolumn{2}{|c|}{-0.0002} & 20.1851 & 0.1601 & -9.0000 & -2.0000 & 0.0000 & 0.0000 \\
\hline 1 & 3 & 3 & 1 & \multicolumn{2}{|c|}{0.0002} & 80.0000 & -1.5000 & -4.4848 & -2.0000 & 0.0000 & 0.0000 \\
\hline 3 & 1 & 3 & 3 & \multicolumn{2}{|c|}{-0.1583} & 20.0000 & 1.5000 & -9.0000 & -2.0000 & 0.0000 & 0.0000 \\
\hline 1 & 1 & 1 & 7 & \multicolumn{2}{|c|}{-0.3232} & 14.3871 & 0.1823 & -9.8682 & -1.7255 & 0.0000 & 0.0000 \\
\hline 7 & 1 & 1 & 7 & \multicolumn{2}{|c|}{-0.1452} & 50.0000 & -0.191 & -8.0773 & -1.7255 & 0.0000 & 0.0000 \\
\hline 0 & 1 & 7 & 0 & 4.00 & & 45.8264 & 0.900 & -4.0000 & 0.0000 & 0.0000 & 0.0000 \\
\hline 0 & 7 & 7 & 0 & 4.00 & & 45.8264 & 0.900 & -4.0000 & 0.0000 & 0.0000 & 0.0000 \\
\hline 2 & 1 & 3 & 7 & -1.50 & & 18.9285 & 0.364 & -6.1208 & 0.0000 & 0.0000 & 0.0000 \\
\hline 2 & 3 & 7 & 3 & 1.50 & & -1.0000 & 0.257 & -6.2100 & 0.0000 & 0.0000 & 0.0000 \\
\hline 1 & 3 & 7 & 3 & -1.43 & & -0.8700 & 0.986 & -2.5424 & 0.0000 & 0.0000 & 0.0000 \\
\hline 7 & 3 & 7 & 3 & -1.50 & & 21.5086 & -1.000 & -4.8869 & 0.0000 & 0.0000 & 0.0000 \\
\hline 2 & 1 & 3 & 9 & 0.17 & & 69.9743 & 0.917 & -7.9557 & 0.0000 & 0.0000 & 0.0000 \\
\hline 1 & 1 & 3 & 9 & 0.25 & & 76.5218 & 1.000 & -2.5503 & 0.0000 & 0.0000 & 0.0000 \\
\hline 3 & 1 & 3 & 9 & -0.25 & & 50.5929 & -0.250 & -6.9285 & 0.0000 & 0.0000 & 0.0000 \\
\hline 2 & 3 & 9 & 3 & -0.25 & & 0.0100 & -0.500 & -4.6984 & 0.0000 & 0.0000 & 0.0000 \\
\hline 1 & 1 & 1 & 11 & 0.50 & & 0.1000 & 0.468 & -11.5274 & -1.7255 & 0.0000 & 0.0000 \\
\hline 2 & 1 & 1 & 11 & 0.00 & & 49.3871 & 0.200 & $0 \quad-10.5765$ & -1.7255 & 0.0000 & 0.0000 \\
\hline 11 & 1 & 1 & 11 & -0.50 & & 95.4727 & -0.208 & $0 \quad-4.8579$ & -1.7255 & 0.0000 & 0.0000 \\
\hline 0 & 1 & 11 & 0 & 4.00 & & 45.8264 & 0.900 & -4.0000 & 0.0000 & 0.0000 & 0.0000 \\
\hline 0 & 11 & 11 & 0 & 4.00 & 00 & 45.8264 & 0.889 & -4.0000 & 0.0000 & 0.0000 & 0.0000 \\
\hline 2 & 1 & 4 & 9 & 0.17 & 14 & 69.9743 & 0.917 & -7.9557 & 0.0000 & 0.0000 & 0.0000 \\
\hline 1 & 1 & 4 & 9 & 0.25 & & 76.5218 & 1.000 & -2.5503 & 0.0000 & 0.0000 & 0.0000 \\
\hline 3 & 1 & 4 & 9 & -0.25 & & 50.5929 & -0.250 & -6.9285 & 0.0000 & 0.0000 & 0.0000 \\
\hline 4 & 1 & 4 & 9 & -0.25 & 00 & 50.5929 & -0.250 & -6.9285 & 0.0000 & 0.0000 & 0.0000 \\
\hline 4 & 1 & 3 & 9 & -0.25 & & 50.5929 & -0.250 & -6.9285 & 0.0000 & 0.0000 & 0.0000 \\
\hline 2 & 4 & 9 & 3 & -0.25 & & 0.0100 & -0.500 & -4.6984 & 0.0000 & 0.0000 & 0.0000 \\
\hline 2 & 4 & 9 & 4 & -0.25 & & 0.0100 & -0.500 & -4.6984 & 0.0000 & 0.0000 & 0.0000 \\
\hline 2 & 3 & 12 & 12 & 0.00 & & 0.0100 & 0.010 & -5.0000 & 0.0000 & 0.0000 & 0.0000 \\
\hline 2 & 3 & 3 & 12 & 0.00 & & 0.0000 & 0.000 & -5.0000 & 0.0000 & 0.0000 & 0.0000 \\
\hline 12 & 3 & 3 & 12 & 0.00 & 00 & 0.0000 & 0.000 & -5.0000 & 0.0000 & 0.0000 & 0.0000 \\
\hline 9 & & ! $\mathrm{N}$ & & of hydr & & & & at 3; Rhb; Deh & & & \\
\hline 3 & 2 & 3 & & 2.1200 & -3 & .5800 & 1.4500 & 19.5000 & & & \\
\hline 3 & 2 & 4 & & 2.1449 & & .5000 & 1.4500 & 19.5000 & & & \\
\hline 4 & 2 & 3 & & 2.2817 & & .8100 & 1.4500 & 19.5000 & & & \\
\hline 4 & 2 & 4 & & 1.9380 & -2 & .5622 & 1.4500 & 19.5000 & & & \\
\hline 3 & 2 & 5 & & 1.5000 & & .0000 & 1.4500 & 19.5000 & & & \\
\hline 4 & 2 & 5 & & 1.5000 & -2 & .0000 & 1.4500 & 19.5000 & & & \\
\hline 5 & 2 & 3 & & 1.5000 & & .0000 & 1.4500 & 19.5000 & & & \\
\hline 5 & 2 & 4 & & 1.5000 & & .0000 & 1.4500 & 19.5000 & & & \\
\hline 5 & 2 & 5 & & 1.5000 & -2 & .0000 & 1.4500 & 19.5000 & & & \\
\hline
\end{tabular}




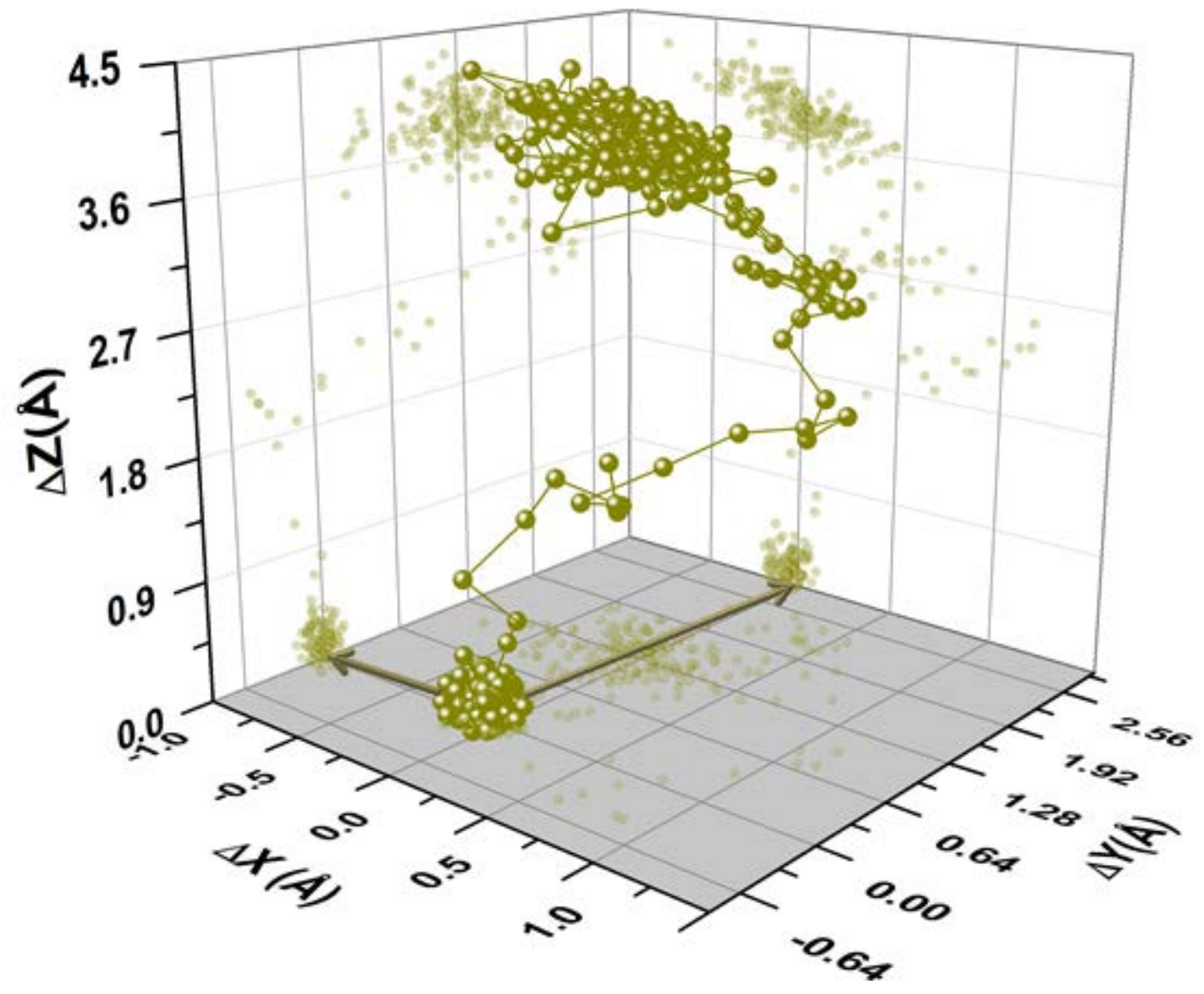

Figure 3S. Distance travelled by the Au adatom during the whole simulation time. The initial position has been placed in the origin of the reference system. Projections (color=light olive green) on the different planes, namely $X Y, X Z$ and $Y Z$, are displayed in order to give an idea of the relative positions of the atom. All the other gold atoms of the interface should be, in principle, in the $\mathrm{XY}$ plane (grey area). 


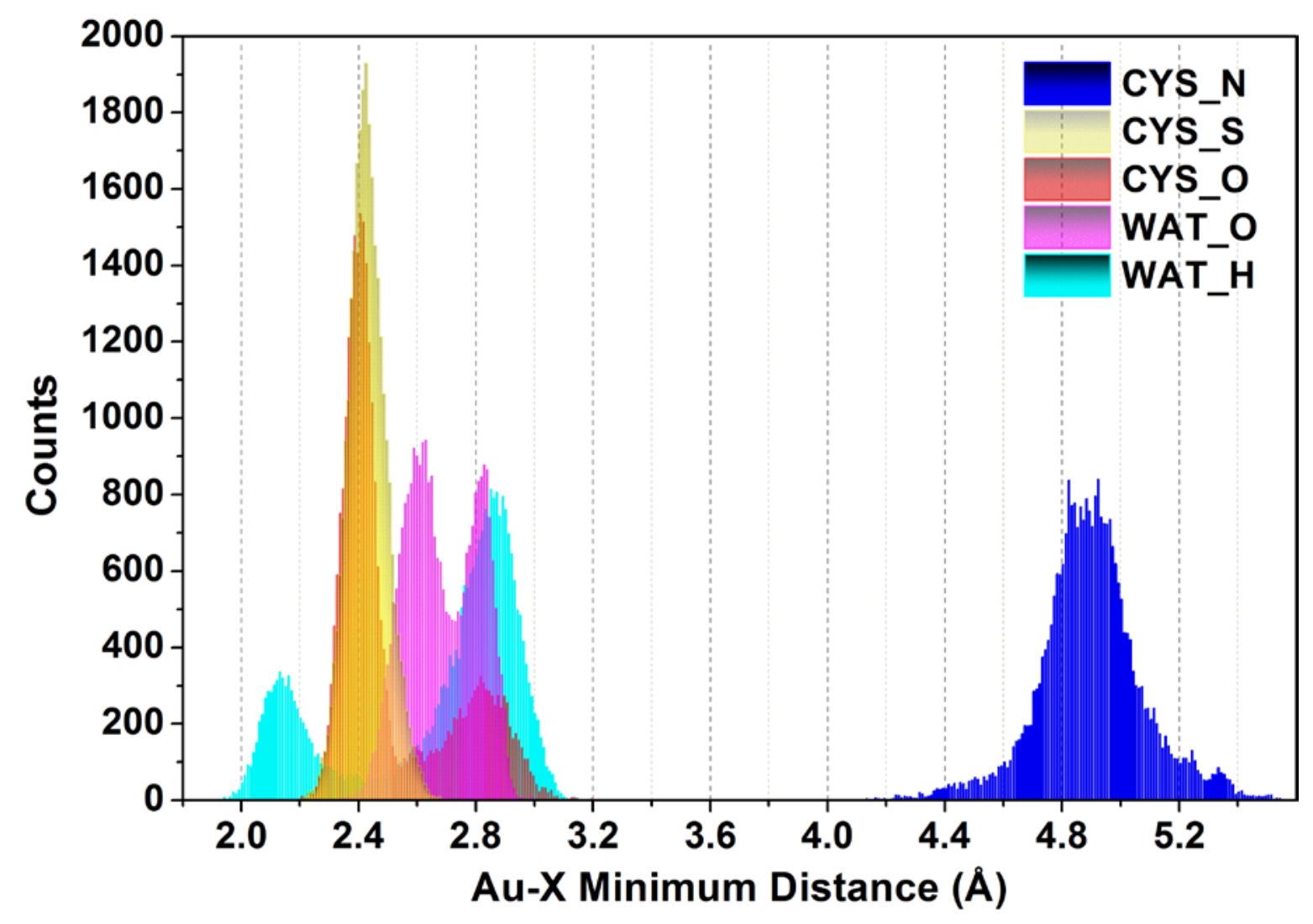

Figure 4S. Distributions of the minimum distances of cysteine and water atoms to the atoms of the support. The amino group remains far from the surface during the whole simulations time, whereas both the oxygens and sulphur atoms of the molecule are located at around $2.4 \AA$ from the slab. Water hydrogens are closer to gold than all the other atoms, whilst water oxygens are found at a longer separation in relation to the carboxyls' ones. 


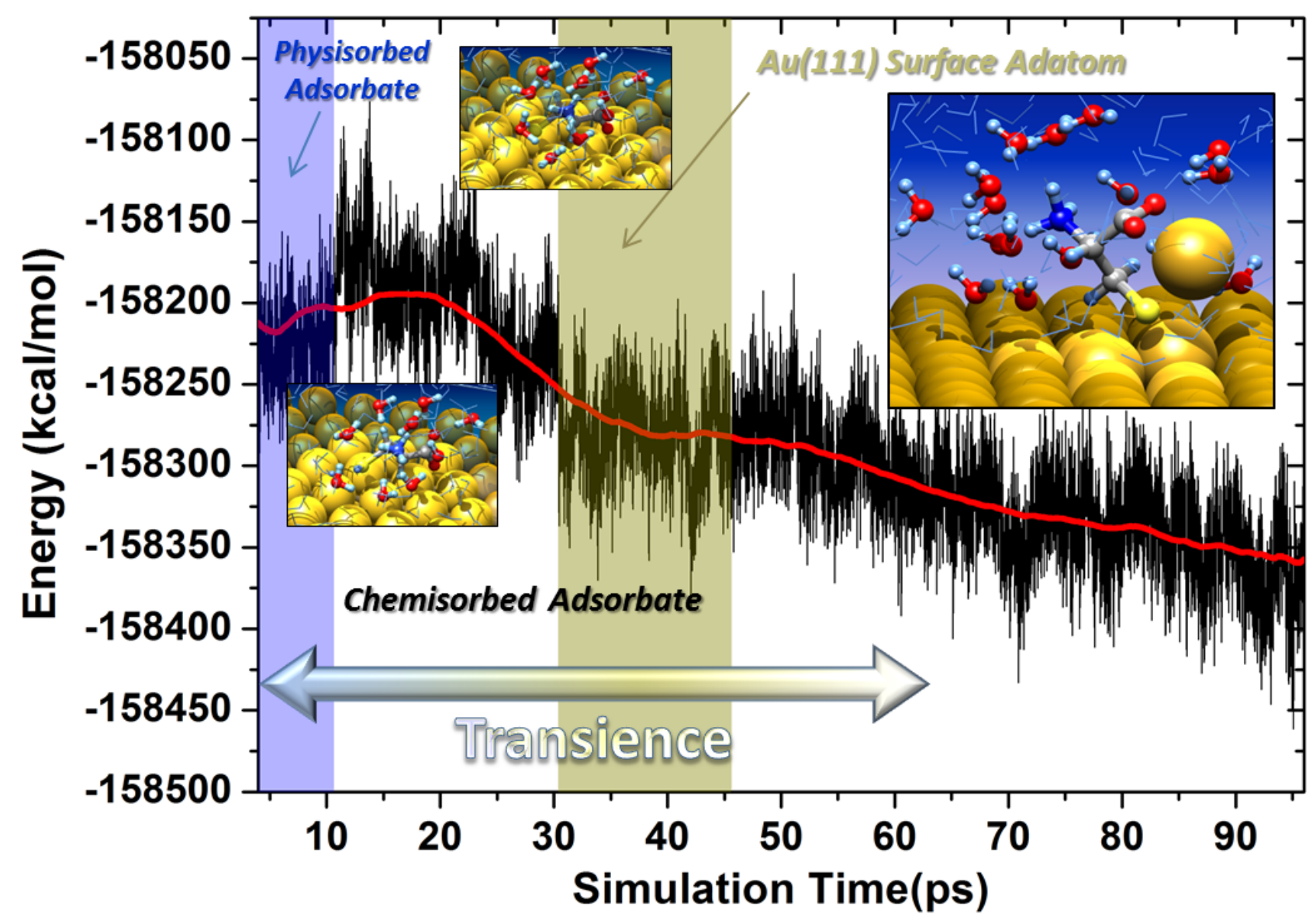

Figure 5S. Potential energy of the system as a function of the simulation time. The smoothing profile (red curve) can be useful to identify the different energy stabilizations during the overall decrease. Initially, CYS is physisorbed on the "perfect" Au(111) surface (light blue section) then it becomes chemisorbed on it (adjacent time zone which cover the rest of the simulation time). After the SH cleavage the energy increases slightly due to the change of binding mode and to the time needed by the system to restore an equilibrium configuration (surrounding solvent arrangement, included). This is reached in the subsequent region between 22 and 30 ps, where the total energy decreases and seems to stabilize around $-158240 \mathrm{kcal} / \mathrm{mol}$. However, at about $30 \mathrm{ps}$ an Au atom moves on top of the surface (in about $0.15 \mathrm{ps}$ ) and try to find the best arrangement there together with the adsorbed molecule (subsequent continuous energy decrease). Finally, the gold atom is "captured" by the sulfur and carboxyl oxygen atoms of the adsorbate and kept between them till the end of the simulation (stabilization of the whole system lower energy). 